\title{
6 Was bedeutet die Praxiszertifizierung?
}

Julia Bellabarba

„Unter Zertifizierung versteht man ein Verfahren, in dem ein (unparteiischer) Dritter schriftlich bestätigt, dass ein Produkt, Prozess oder Dienstleistung mit festgelegten Anforderungen konform ist.“ (aus: DIN EN ISO 45020:1998-07)

Eine Zertifizierung der niedergelassenen Vertragsärzte und -psychotherapeuten erfolgt derzeit freiwillig. Jeder Praxisinhaber kann selbst entscheiden, ob er eine Zertifizierung seines QM-Systems anstreben möchte. Die Zertifizierung erfolgt durch unabhängige, akkreditierte Partner des jeweiligen QM-Systems, das in der Praxis Anwendung findet. D. h. eine Zertifizierung ist nur möglich, wenn die Praxis ein zertifizierungsfähiges QM-System umgesetzt hat. Die Zertifizierungsgesellschaften sind eigenständige Gesellschaften, die sich für die Zertifizierung eines Systems qualifiziert haben, sie sind jedoch weder mit den Kostenträgern noch mit den Ärztevertretungen oder ähnlichen Organisationen verwoben.

Es liegen keine Zahlen für alle QM-Systeme vor, $\mathrm{QEP}^{\circledR}$ hat jedoch einen gewissen run zu verzeichnen: Es haben sich 187 Praxen nach diesem System zertifizieren lassen (Stand 05/09). 2007 führten die Kassenärztlichen Vereinigungen eine repräsentativen Umfrage durch: 1.757 Niedergelassene aus einer Zufallsstichprobe machten freiwillig Angaben zur Zusatzfrage, welches QM-System sie in ihrer Praxis umgesetzt haben. Der Umfrage zufolge ist $\mathrm{QEP}^{\circledR}$ das am häufigsten verwendete QM-System in Praxen (36\%). ISO 9oo ist mit 27,9\% am zweithäufigsten vertreten, KPQM wurde von 3,1\% der Ärzte angegeben (Pressemitteilung der KBV 10.10.2008).

Die Praxis, die sich nicht zertifizieren lassen möchte, kann die Vorgaben der QM-Richtlinie des GBAs umsetzen und somit den gesetzlichen Anforderungen entsprechen, ohne auf eines der genannten Systeme zurückzugreifen 
und ohne sich zertifizieren zu lassen. Soweit die Rechtslage. Für einige Fachgruppen liegen allerdings schon jetzt Verpflichtungen zur Zertifizierung im Rahmen bestimmter Verträge mit Kostenträgern vor (beispielsweise im Rahmen des VdEK Vertrags mit den Hausärzten in Hessen). Wie bereits in Kapitel I.3 erwähnt, ist diese Tendenz problematisch, da es keine Transparenz über den Markt der Zertifizierungen gibt und keine neutrale Akkreditierungsstelle, die über die Güte der Zertifikate befindet. Darüber hinaus ist (irgend-)ein QM Zertifikat nicht unbedingt ein valider Indikator für die Güte einer Praxis.

Die Zertifizierung einer Praxis bildet nicht den Abschluss kontinuierlicher Prozessoptimierung, sondern sollte für die Praxis die Bedeutung eines Meilensteins haben. Das (tägliche) systematische Streben, Qualität zu halten und zu verbessern, und Qualitätsmanagement als rekursiven fortwährenden Prozess zu begreifen, kann durch eine externe Prüfung dokumentiert werden, drückt sich jedoch in erster Linie durch die (Selbst-)Verpflichtung zur Selbstbewertung aus.

Es lohnt sich, im Team oder mit Kollegen über Sinn und Unsinn eines Zertifikats zu diskutieren, da an das Zertifikat in der Regel bestimmte strategische Entscheidungen gebunden sind (beispielsweise Verträgen zur Integrierten Versorgung beizutreten) und in einer solchen Diskussion Qualitätsziele der Praxis explizit gemeinsam formuliert werden können.

\section{Aufgabe 4 für anwendungsfreudige Leser}

Besprechen Sie mit Ihren Mitarbeiterinnen oder mit Ihren Kollegen, welche Vor- und Nachteile eine Zertifizierung für Ihre Organisation bringen würde, und ergänzen oder verändern Sie die hier aufgeführte Pro- und Contra-Liste (s. Tab. 5).

\section{Tab. 5 Pro und Contra Zertifizierung}

\begin{tabular}{|c|c|}
\hline Pro & Contra \\
\hline $\begin{array}{l}\text { Die Zertifizierung: } \\
\text { - } \quad \text { veranlasst zu einer gründlichen } \\
\text { Überarbeitung und Verbesserung aller } \\
\text { relevanten Prozesse. } \\
\text { - } \quad \text { ergänzt die Selbstbewertung und vermittelt } \\
\text { Aufschluss über Schwachstellen. } \\
\text { - verstärkt den Teamgeist. } \\
\text { - } \text { macht stolz. } \\
\text { - } \text { kann Patientenwahrnehmung positiv } \\
\text { beeinflussen. } \\
\text { - } \text { bietet Sicherheit in Haftungsfragen. } \\
\text { - } \text { kann strategisch sinnvoll sein. } \\
\text { - kann Konkurrenzvorteile haben. } \\
\text { - } \text { kann langfristig Vergütungsvorteile } \\
\text { bewirken. }\end{array}$ & $\begin{array}{l}\text { Die Zertifizierung: } \\
\text { - } \text { kostet Geld. } \\
\text { - } \text { kostet Zeit. } \\
\text { - } \text { kostet Nerven. } \\
\text { - } \text { kann zu Bürokratisierung beitragen. } \\
\text { - } \text { kann Konflikte im Team verschärfen. } \\
\text { - } \text { nützt nichts, wenn sie nur „Pro-Forma“ } \\
\text { erfolgt. } \\
\text { - } \text { kann dazu beitragen, dass sich Praxen an } \\
\text { Formalismen „aufhängen“. } \\
\text { - } \text { kann man einfach aus prinzipiellen Gründen } \\
\text { ablehnen (,In meiner Praxis hat kein Auditor } \\
\text { etwas zu suchen!“). }\end{array}$ \\
\hline
\end{tabular}


Pro Contra

- kann der Vermeidung von Sanktionen dienen (\$ 9 GBA-Richtlinie).

- bietet Verhandlungsvorteile gegenüber Kreditinstituten (BASEL) und Versicherungen.

Wenn ein Team oder eine Organisation eine Praxiszertifizierung in Erwägung ziehen, ist die folgende Checkliste (s. Tab. 6) hilfreich:

Tab. 6 Checkliste für Praxen, die eine Zertifizierung planen

Zertifizierung: Checkliste für Praxen

Bietet das QM-System, das wir haben, die Möglichkeit zur Zertifizierung?

Welche Ziele wollen wir durch eine Zertifizierung erreichen?

Welche Strategie verfolgen wir damit in Hinblick auf Kooperationspartner und Kostenträger?

Gibt es zu diesem Thema Konsens im Team und in der Praxisleitung?

Wieviel Zeit und Geld wollen/können wir für die Zertifizierung einplanen?

Welchen zeitlichen Rahmen wollen wir uns für das Projekt QM-Zertifizierung setzen?

Wer ist für die Einhaltung des zeitlichen Rahmens zuständig?

Planen wir externe Moderation/Beratung ein?

Häufig verwendete Begriffe im Zusammenhang mit einer QM-Zertifizierung:

- Akkreditierung: offizielle Anerkennung und Lizenz einer Zertifizierungsgesellschaft, ein QM-System zertifizieren zu dürfen.

- Audit $=$ Visitation $=$ Assessment: die eigentliche Begutachtung (Begehung) der Praxis durch einen Visitor (= Auditor) vor Ort.

- Fremd- und Selbstbewertung: die regelmäßige Selbstbewertung der Praxis ist in der CBA-Richtlinie vorgesehen, Fremdbewertungen erfolgen i.d. R. im Rahmen einer Zertifizierung.

- Zertifizierungsgesellschaft: unabhängige, unparteiische Organisation, die über die Kompetenz und Lizenz verfügt, die Umsetzung eines QMSystems in einer Organisation zu überprüfen.

- Zertifizierung: erfolgt in der Regel durch Prüfung des QM-Handbuchs, durch Begehung (Audit) und durch Befragung der Leitung und der Mitarbeiter. Das (externe) Zertifizierungsaudit wird durch eine neutrale Zertifizierungsgesellschaft durchgeführt.

„Dabei auditiert die neutrale Zertifızierungsstelle das Qualitätsmanagementsystem eines Unternehmens auf dessen Auftrag und vergibt bei Erfüllung der Anforderungen (...) ein Zertifikat. (...) Die formale Kompetenz, Unabhängigkeit und Integrität der Zertifizierungsstellen leitet sich aus deren Akkreditierung bei einer übergeordneten Trägergemeinschaft für Akkreditierung ab“ (Kamiske u. Brauer 1999) 


\section{Literatur}

Bellabarba J: Von der Philosophie über die Strategie zur Methode: Systematisches umfassendes Qualitätsmanagement in einer Arztpraxis für Kinder- und Jugendpsychiatrie und -psychotherapie. Forum für Kinderund Jugendpsychiatrie, Psychosomatik und Psychotherapie 2007; 2: 7-24

Birkner B: Zertifizierung einer gastroenterologischen Gemeinschaftspraxis nach DIN ISO EN 9001 - vernetzt mit den Leitlinien einer wissenschaftlich-medizinischen Fachgesellschaft. ZaeFQ 2000; 94: 639-643

Kamiske G, Brauer JP: Qualitätsmanagement von A-Z. 6. Aufl. München: Hanser Verlag 2007

\section{links}

www.benchmarking-qm.de

www.kbv.de/themen/9042.html

Glossar auch unter: www.kvb.de/servlet/PB/show/1116432/QM-Richtlinienbroschuere_4_2008.pdf 